\title{
Comportement mécanique des prothèses totales de hanche au pic de chargement
}

\author{
Atef Boulila ${ }^{1, a}$, Khemaïes Jendoubi ${ }^{1,2}$, Ali Zghal ${ }^{2}$, Mustapha Khadhraoui ${ }^{3}$ \\ ET PATRICK CHABRAND ${ }^{4}$ \\ 1 Laboratoire de Biomécanique Orthopédique (LBO) INO. M.T. Kassab, Ksar Saïd, 2010 Manouba, Tunisie \\ 2 Laboratoire de Mécanique des Solides, des Structures et de Développement Technologique, ESST - Tunis, \\ BP 56, Beb Mnara, 1008 Tunis, Tunisie \\ 3 Université de Provence/UNIMECA, Technopôle de Château Gombert, 60 rue F. Joliot Curie, \\ 13453 Marseille Cedex 13, France \\ 4 Laboratoire LABM UMSR, Université de la Méditerranée, 163 avenue de Luminy, case 918, 13288 Marseille, France
}

Reçu le 11 septembre 2007, accepté le 18 décembre 2008

\begin{abstract}
Résumé - En dépit des progrès enregistrés en Arthroplastie Totale de Hanche (ATH), sous certaines conditions, les contraintes prothétiques peuvent être amplifiées et transmises aux ancrages osseux avec un risque accru de descellement (B. The et al., J. Biomech. 41 (2008) 100-105). Les pics de chargement sont identifiés parmi les facteurs qui conduisent aux échecs des ATH (A. Boulila, Optimisation et aide à la conception d'implants orthopédiques - Étude de l'usure d'implants articulaires de hanche, Travaux non publiés et Rapport d'avancement, INO MT Kassab, décembre 2006, Tunis, 82 p.). Cette étude porte sur une simulation numérique des prothèses de hanche hybrides, de géométrie et de couple de matériaux différents. On montre que certaines géométries $(\emptyset 28 \times 56 \mathrm{~mm})$ et certains couples de matériaux (alumine/polyéthylène), peuvent atténuer les contraintes induites après choc et protéger la prothèse contre l'usure ou les ruptures prématurées. Devant une panoplie d'une cinquantaine de modèles de prothèses de hanche existant sur le marché (M.R. Urist, Curr. Prob. Surge. 12 (1975) 1-54), certaines PTH de diamètre (interne et externe) incompatible avec un niveau de contraintes admissible doivent être éliminées. Une combinaison judicieuse des matériaux de contact pour favoriser l'amortissement (cas du couple polyéthylène/céramique) peut être une solution pour absorber les chocs et remédier au problème de descellement.
\end{abstract}

Mots clés : Prothèse / hanche / polyéthylène UHMWPE / inox 316L / céramique / surcharge / élément-finis

\begin{abstract}
Mechanical behaviour of the total hip prostheses to the loading peak. Under severe conditions, the prosthetic stresses in Total Hip Arthroplasty (THA) can be amplified and transmitted to bone anchoring. This increases the risk of loosening (B. The et al., J. Biomech. 41 (2008) 100-105). The loading peaks are identified as the factors which lead to the failures of the THA (A. Boulila, Optimisation et aide à la conception d'implants orthopédiques - Étude de l'usure d'implants articulaires de hanche, Travaux non publiés et Rapport d'avancement, INO MT Kassab, décembre 2006, Tunis, 82 p.). This study deals with a numerical simulation of the hybrid hip prostheses which have a different geometry and some materials friction. It is shown that some geometries $(\emptyset 28 \times 56 \mathrm{~mm})$ and friction materials (alumina/polyethylene), can attenuate the stresses induced after a peak and protect the prosthesis against the premature wear or fractures. In front of panoply of about fifty models of hip prostheses existing on the market (M.R. Urist, Curr. Prob. Surg. 12 (1975) 1-54), some THP diameters (intern and external) are incompatible with a
\end{abstract}

${ }^{a}$ Auteur pour correspondance : atef.boulila@laposte.net 
working level of stresses and must be eliminated. A judicious combination of friction materials to support damping (case of the polyethylene/ceramic) can be a solution to absorb the shocks and to cure the problem of loosening.

Key words: Prostheses / hip / polyethylene UHMWPE / AISI 316L SS / ceramics / overload / finite-element

\section{Introduction}

En élasticité, la déformation des structures simples (barre, poutre, ...) sous chargement donné, dépend de l'aire de sa section mais surtout des moments quadratiques des sections. Pour les structures complexes telles que les prothèses articulaires (de hanche, de genou, ...), la limite d'efficacité de ces implants dépend de plusieurs paramètres entre autre le type de chargement appliqué et leurs géométries [4].

Plusieurs études relevant de la biomécanique orthopédique, montrent qu'un bon design de la prothèse à travers une meilleure optimisation des facteurs de forme (diamètre, épaisseur, ...) peut améliorer la durée de vie des implants articulaires, d'où la tendance de concevoir des prothèses (PTH, PTG, ...) de plus en plus anatomiques [5].

Dans la pratique, les facteurs de forme associés à ces structures sont limités par des contraintes de fabrication (formes complexes et congruentes) mais aussi par la nature des mouvements relatifs à transmettre (rotation ou rotation plus glissement). Sous l'effet d'un chargement prothétique élevé, à prédominance triaxiale, en milieu biologique, la défaillance d'un implant articulaire peut faire l'objet d'une superposition de plusieurs modes d'endommagement avant la ruine totale de la prothèse [6] où le recours à une arthroplastie de reprise est alors envisageable.

Dans la recherche des performances des implants articulaires, théoriquement on choisit des couples de matériaux bien élaborés et une géométrie à surface portante élevée pour diminuer les pressions de contact comme on l'observe sur les prothèses de hanche céphaliques où le diamètre de la tête peut dépasser les 40 ou $50 \mathrm{~mm}$ [6]. Mais en faisant cela, on pousse la structure (prothèse) vers des conditions tribologiques plus sévères par une augmentation des frottements et une activation du phénomène d'usure [7].

Selon le type de matériaux de friction utilisés en chirurgie orthopédique (polyéthylène (UHMWPE), acier inoxydable $(316 \mathrm{~L})$, titane $\left(\mathrm{Ti}_{6} \mathrm{Al}_{4} \mathrm{~V}\right)$, céramique $\left(\mathrm{Al}_{2} \mathrm{O}_{3}\right)$ [5,6]), les endommagements des prothèses, sous chargement, peuvent apparaitre sous forme de déformations plastiques (cas du polyéthylène, ...) et de rupture instable (cas de la céramique) mais le plus souvent, sous chargement cyclique ils sont dus à la fatigue, fatigue-fluage, corrosion, fatigue-corrosion et évidemment l'usure sous ses différents aspects $[7,8]$.

La variabilité des paramètres anatomophysiologiques d'un sujet à un autre, ne facilite pas une standardisation (normes) systématique de ces composants, conçus pour travailler en milieu sévère pour de longues durées [9].

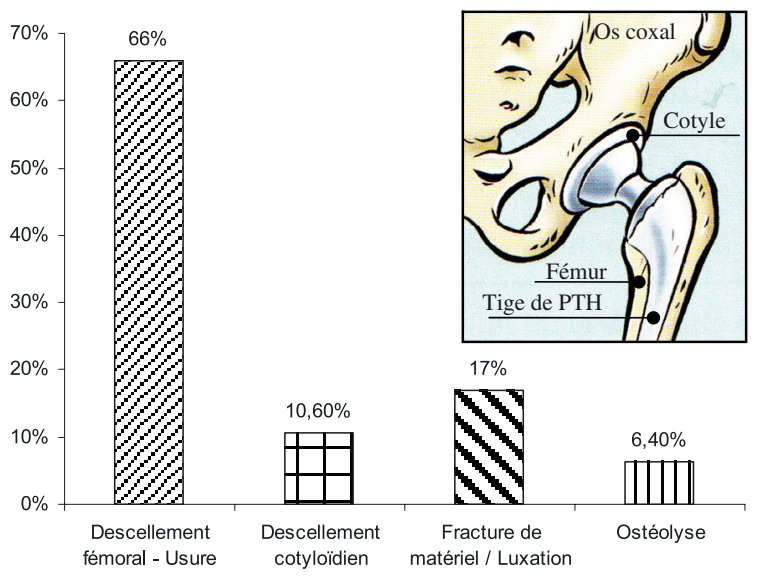

Fig. 1. Arthroplastie totale de hanche (a), causes de reprise de PTH (b) (Registres Institut M.T. Kassab) [4].

Une prothèse articulaire de hanche fonctionnelle, exige des ajustements rigoureux des géométries (diamètre, épaisseurs, ...), des matériaux de friction élaborés, un mode d'implantation justifié (avec ou sans ciment) et des contraintes prothétiques bien optimisées.

Les principales causes des défaillances des arthroplasties totales de hanche sont dues à des descellements de la tige fémorale ou l'usure de la composante acétabulaire de PTH [10]. Selon une étude rétrospective menée à l'Institut M.T. Kassab sur des dossiers traités de 2002 à 2007 [11], $66 \%$ des cas de défaillances sont dus au descellement et à l'usure, 6,3\% d'ostéolyse (maladie osseuse), 10,6 \% des descellements cotyloïdiens. La luxation et les fractures de matériels représentent un taux global de $17 \%$. Selon l'étiologie de la population concernée ces défaillances (fractures et luxations) sont dues principalement aux surcharges excessives appliquées à la prothèse.

\section{Approche biomécanique de l'articulation coxo-fémorale}

\subsection{Mobilité de la hanche}

La stabilité du squelette humain, dépend en grande partie, de la persistance et la qualité fonctionnelle de l'articulation coxo-fémorale. Sur le plan biomécanique, on distingue trois degrés de liberté activés autour de trois axes propres à l'articulation de la hanche :

- axe antéropostérieur, assurant l'abduction et l'adduction $(\mathrm{AB} / \mathrm{AD})$; 


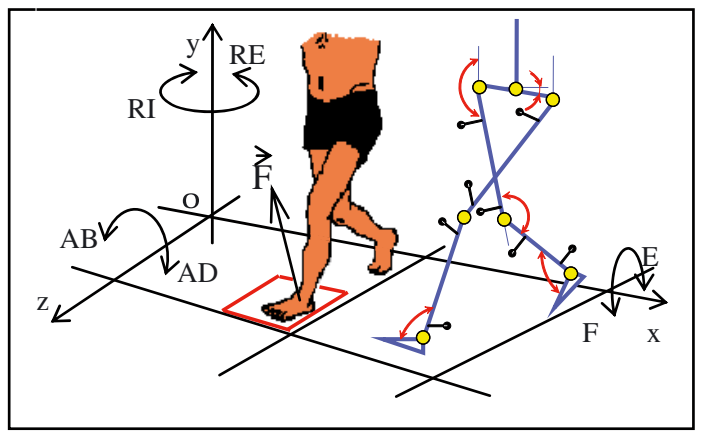

Fig. 2. Résultante transmise à la hanche en position d'appui monopodal.

- axe vertical confondu avec l'axe longitudinal du membre inférieur, qui permet les mouvements de rotation externe et rotation interne (RI/RE);

- axe transversal, autour duquel s'effectuent la flexion et l'extension $(\mathrm{F} / \mathrm{E})$.

Les amplitudes maximales de ces angulations dépendent de l'état physio-neurologique du patient. Selon Calonius et al. [12], ces angles sont définis par $\theta_{1} \pm 6^{\circ}$ pour $l^{\prime}(\mathrm{AB} / \mathrm{AD}), \theta_{2} \pm 6^{\circ}$ pour la $(\mathrm{RI} / \mathrm{RE})$ et $\theta_{3} \pm 23^{\circ}$ pour la $(\mathrm{F} / \mathrm{E})$.

\subsection{Chargement et pression appliqués à la hanche}

Plusieurs auteurs ont cherché à évaluer les contraintes supportées par la tête du fémur en utilisant les principes de la biomécanique articulaire. Parmi ces auteurs on cite Pauwels [13], Rydell [14] et Paul [15].

\section{- Modèle analytique}

Il s'agit d'un calcul développé par Pauwels [13] sur calques radiologiques par projection dans le plan frontal $(o, \vec{x}, \vec{y})$ (Fig. 3), alors que l'axe du col fémoral est antéversé, ce qui devait tenir compte du plaquage des tendons antérieurs (ilio-psoas et droit fémoral) et ce d'autant plus que l'antéversion du col est plus marquée. Pour ce que l'on nomme «Balance de Pauwels » [13] (Fig. 3), la distribution des efforts se caractérise par :

- en appui monopodal, le poids du corps en charge est celui du corps moins le poids du membre inférieur portant;

- l'action équilibratrice est centrée sur le moyen fessier, et plus spécialement sur sa fibre moyenne. Le vecteur est toujours dirigé obliquement en bas et en dehors;

- la résultante est construite à partir des deux vecteurs précédents. Elle est oblique en bas et en dehors, à environ $16^{\circ}$ sur la verticale [13] et passant par le centre de la tête fémorale;

- le point de balance, de part et d'autre duquel les forces en présence doivent s'équilibrer, est présenté par le centre de la tête fémorale;

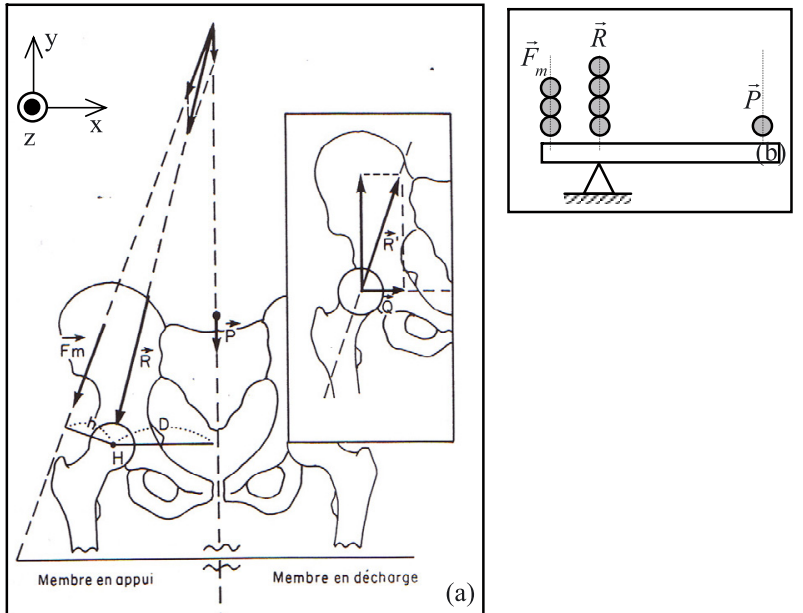

Fig. 3. Modèle anatomique (a), modèle de calcul «balance de Pauwels » : le bras de levier gravitaire est 3 fois plus grand que celui du moyen fessier, d'où $R=4 P$ (b).

- les bras de levier sont $(D)$ pour la force gravitaire (poids : $P$ ), environ trois fois plus important que celui (h) de la force musculaire (action des moyens fessiers $\left.F_{\mathrm{m}}\right)$;

- il en résulte que, pour qu'il y ait équilibre, il faut que les moments des forces concentrées soient égaux, autrement dit $P D=F_{\mathrm{m}} h$. Sachant que les mesures anatomiques sont définies par $D=3 h$, d'où $F_{\mathrm{m}}=3 P$. Au total, la résultante $R$ est égale à la somme algébrique des deux forces, soit $R=4 P$.

\section{- Technique expérimentale}

Le modèle de Rydell [14] fait appel à l'extensométrie pour la mise en place de jauges de déformation sur une hanche de cadavre. Les résultats ont été rapportés par Carlson [16]. Les données sont donc plus fiables, encore qu'elles ne peuvent être identiques à une situation physiologique véritable. Ses résultats sont les suivants : en appui bipodal : $R=1 / 3$ de $P$; en appui monopodal : $R=2,5$ à $3 P$; en appui monopodal, pied au contact du sol : $R=1 / 8 P$; en appui monopodal, hanche et genou fléchis (donc sans appui) : $R=1 / 8$ de $P$.

L'autre technique expérimentale de Paul [15,17] fait appel à une plate-forme munie de jauges d'extensométrie permettant de mesurer la force de réaction du sol sur le pied dans les trois plans de l'espace, ainsi que les trois moments correspondants. Connaissant la position des segments osseux et les angles articulaires, il est possible d'évaluer les contraintes transmises à travers les articulations. Les muscles sont regroupés par fonction et leurs forces sont évaluées par référence à la section totale. L'analyse du comportement des ligaments est faite grâce à l'anatomie et à des radiographies. Cette technique porte le nom de «dynamique inverse »[18]. L'évolution des chargements appliqués à la liaison coxo-fémorale, selon les principes de la biomécanique se résume ainsi :

- marche normale : maximum 5 à $6 P$, pendant des durées inférieures au 1/10 de seconde; 


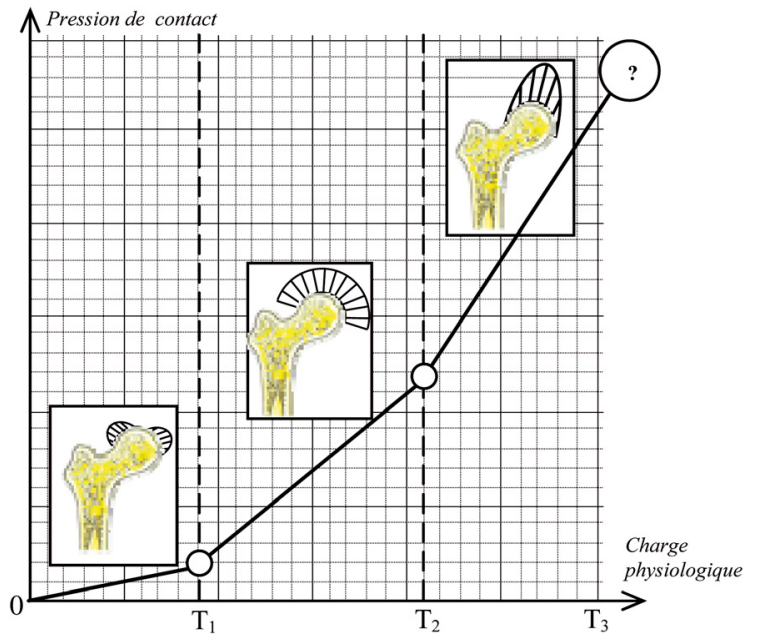

Fig. 4. Évolution de la pression de contact sur la tête fémorale en fonction de la charge.

- marche à vitesse rapide : maximum de 6,2 à $7,5 P$, pendant des durées inférieures au 1/10 de seconde;

- montée d'une rampe de $10 \%$ : maximum de $5 P$;

- descente d'une rampe de $10 \%$ : maximum de $6 P$.

Paul et al. [17] insistent beaucoup sur la variation quasiinstantanée de ces valeurs, ce qui favorise la lubrification de l'articulation et la nutrition du cartilage, chose qui ne pouvait pas se reproduire sur une articulation prothésée et inerte.

Sur le plan biomécanique, il en résulte de l'augmentation de la charge $R$, une augmentation progressive des pressions de contact entre tête de fémur et os coxal (Fig. 4). On y observe que la répartition uniforme des pressions $\left[T_{1}, T_{2}\right]$ ne se fait que dans certaines limites audelà desquelles se manifeste une hyperpression polaire de plus en plus forte pour des chargements sévères $($ choc, ...) $\left[T_{2}, T_{3}\right]$. On rejoint ainsi la théorie de Pauwels sur l'hyperpression polaire (céphalique) quand $R$ devient trop forte comme le cas d'une coxa-valga [6].

L'articulation naturelle de la hanche fonctionne donc non seulement en raison de la forme ogivale de l'architecture et de l'élasticité du cartilage mais aussi grâce à un coefficient de frottement pratiquement nul de la liaison dont on est incapable de reproduire artificiellement (PTH) [19].

\section{3 Étendue de surface de contact de l'articulation naturelle de la hanche}

Pour l'articulation normale de la hanche, les déformations cartilagineuses rendent possible le plein contact du cartilage dans toutes les régions à condition que la charge soit suffisante. Il faut voir là un mécanisme naturel «merveilleux » que nous ne sommes pas capables de réaliser mécaniquement par les articulations artificielles [20]. C'est donc grâce aux déformations du cartilage que l'on peut réellement dire que l'interface chondral est sphérique.

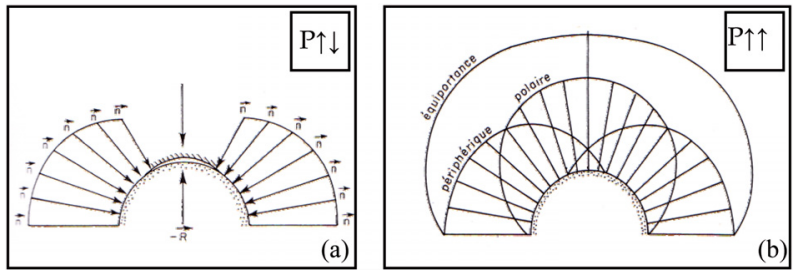

Fig. 5. Pression sur la tête fémorale, $P$ : charge mécanique [3].

Il faut considérer que nous sommes face à une architecture déformable. L'élasticité de chacun des composants, os et cartilage, en est la raison, mais celle du cartilage, mille fois supérieure à celle de l'os souschondral, joue le rôle prédominant. Si on exerce sur un tel ensemble une charge progressivement croissante, dans une première phase, la portance sera essentiellement périphérique et para-équatoriale. On y remarque que les forces se répartissent de chaque côté autour d'un axe oblique, l'ensemble réalisant dans l'espace une sorte de couronne. Chacune des forces est ici une fonction sinusale de la force incidente, conforme au modèle décrit, mais selon une répartition différente.

Accentuons encore la charge tel que le pôle céphalique, non seulement touche le dôme cotylö̈dien, mais encore appuie sur lui assez fortement. On conçoit alors que la congruence devient ici totale. Dans la région du pôle apparaît une répartition polaire semblable à celle du modèle géométrique.

Dans le même ordre d'idée, il est clair que les forces périphériques n'ont pas disparu. Leur répartition se présente selon le même gradient bien que modifiées dans leur valeur absolue. Il se produit en réalité une superposition de deux phases et plus précisément une superposition d'une infinité de phases dont nous devons faire la somme intégrale. Nous obtenons ainsi le type de la figure 5b, qui est la somme vectorielle des deux phases retenues. Elle montre bien la répartition égalitaire obtenue [6] et aussi la particularité de la liaison coxo-fémorale proche d'un système mécaniquement asservi, mais jusqu'à quelle charge peut-il résister?

\section{Matériels et méthodes}

La simulation des surcharges mécaniques des PTH repose sur les modèles géométriques simples de sphéricité supposée «parfaite » et de dimensions semblables à celles trouvées par des études cliniques. Les lois de comportement des matériaux de friction sont du type élastoplastique; le contact entre la tête et le cotyle suit la loi de frottement de Coulomb. Le contact est supposé sec, à jeu uniforme.

Tous les calculs sont faits en Hypothèse des Petites Perturbations (HPP) et développés sur le code Abaqus 6.5 . 

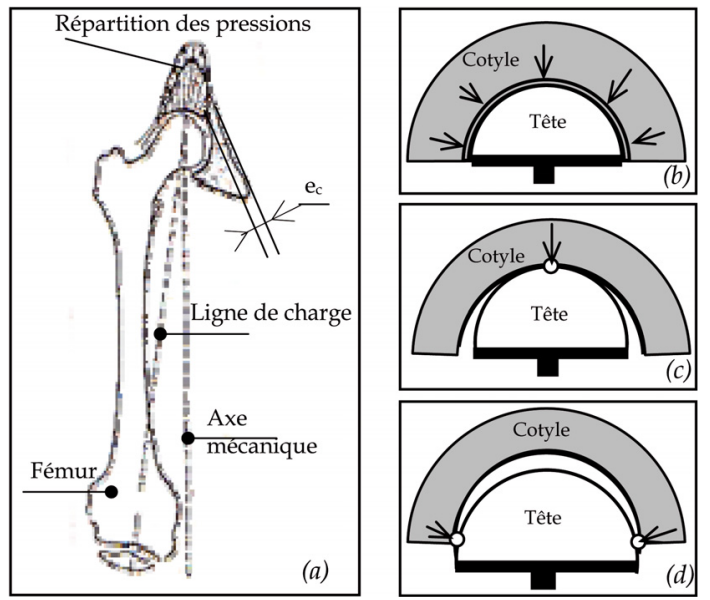

Fig. 6. Articulation coxo-fémorale (a), congruence entre cotyle et tête de PTH (b), congruence entre cotyle et tête : diamètre de tête plus petit que le cotyle (c) diamètre du cotyle plus petit que le diamètre de tête $(\mathrm{d})$.

\subsection{Modèle géométrique équivalent}

Si l'on prend le cas d'une articulation artificielle (PTH), celle-ci n'est pas sphérique. Sur le plan technique, il est impossible de concevoir deux sphères réelles concentriques qui aient un même rayon car alors, elles seraient confondues. Pour qu'un mouvement de rotation soit réalisable, l'un par rapport à l'autre, il faut nécessairement ménager un espace évolutif entre chacune des surfaces. Bien que très petit, celui-ci permet de pallier les écarts de sphéricité d'un usinage « toujours imparfait $»$. Or on sait que le contact de deux sphères géométriques de rayons différents, l'une étant à l'intérieur de l'autre, est un point et qu'il n'y a pas de surface de contact. Ce point ne peut devenir surface qu'en raison de l'élasticité des matériaux constitutifs des deux sphères (polyéthylène, métal ou céramique). Cette élasticité entraîne une déformation de la tête et du cotyle dans la région polaire. Il apparaît là une aire de contact circulaire et aplatie. L'aplatissement de la tête et l'enfoncement correspondant du cotyle aussi petit soit-il, ainsi que les phénomènes de fluage du polyéthylène, font que les centres respectifs de chacun d'eux ne coïncident pas. En conséquence, les régions équatoriales et para-équatoriales n'ont pas de contact. En définitive, nous obtenons un aspect semblable à celui de la figure 6 où l'image est toutefois exagérée et agrandie.

Le modèle géométrique étudié est composé de la tête de PTH et du cotyle (Fig. 7). La tête est montée par adhérence à la tige de $\mathrm{PTH}$, grâce à un cône morse de conicité $5^{\circ} 20^{\prime \prime}$. Cette tige est ancrée dans le fémur avec ou sans ciment acrylique à base de PMMA.

\subsection{Approche analytique}

Plusieurs modèles existant dans la littérature proposent des formulations analytiques capables de
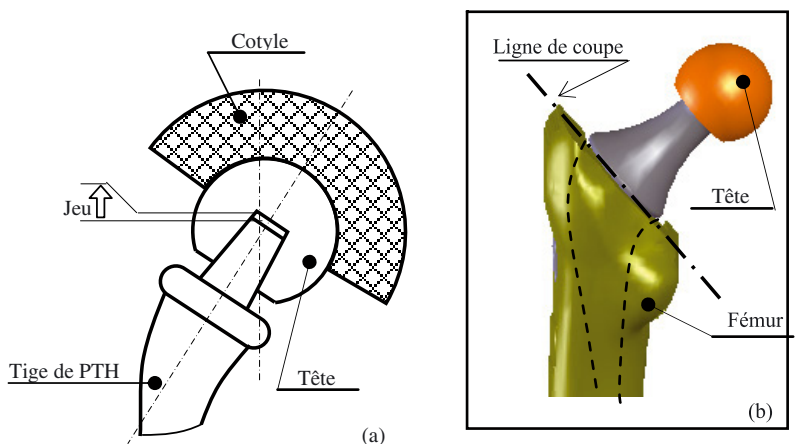

Fig. 7. Prothèse totale de hanche et interface (a), contact tige de PTH - fémur (b).

reproduire la forme de la pression de contact, supposée être élastique [21,22].

Telles qu'ils existent à l'heure actuelle, avec leurs technologies améliorées, les divers modèles de PTH ne diffèrent guère dans leur conception générale des grands classiques qui ont inauguré l'ère de la chirurgie prothétique de la hanche (prothèse de Charnley). Du point de vue tribologique [23], ces mécanismes de transmission de mouvement, devront épouser des formes congruentes adaptables aux liaisons naturelles endommagées. Les chargements physiologiques appliqués au système d'articulations associé à la hanche naturelle ou prothétique dépendent en grande partie de l'état postural du sujet et l'amplitude des mouvements lors de la marche.

Sachant que le contact entre la tête et le cotyle d'une PTH est un emboîtement sphérique et que la charge physiologique est orientée dans le plan frontal d'un angle de $16^{\circ}$ par rapport à la verticale, il est nécessaire d'évaluer cette charge en fonction des différents angles que fait la tête par rapport au cotyle.

Nous admettons que dans le plan frontal, l'équation analytique de la pression de contact (Fig. 8) prend la forme suivante :

$$
p(\theta)=p_{\max }\left(\frac{1+\cos 2 \theta}{2}\right)
$$

où $\left(p_{\max }\right)$ est la pression maximale de contact et $(\theta)$ le déplacement angulaire dans le plan frontal de la tête de PTH par rapport au cotyle. L'angle $\theta$ (arc (ab)) varie entre deux valeurs extrêmes $(0)$ et $\left(\theta_{0}\right)$ (angle critique de non subluxation) et $\varphi$ un angle qui varie entre 0 et $2 \pi$.

La force appliquée à la tête de $\mathrm{PTH}$ peut s'écrire : $\mathrm{d} \vec{R}=p(\theta) \mathrm{d} S \vec{u}$ où $\mathrm{d} S$ représente l'élément de surface, soit : $\mathrm{d} S=R \mathrm{~d} \theta R \sin \varphi \mathrm{d} \varphi$. La pression $p(\theta)$ s'écrit alors :

$$
p(\theta)=\frac{4\|\vec{R}\|}{\pi R^{2}\left(3-2 \cos 2 \theta_{0}-\cos 4 \theta_{0}\right)}(1+\cos 2 \theta)
$$

La valeur maximale de $p(\theta)$ sera utilisée comme condition de chargement dans nos modèles numériques.

L'élasticité et la bio-fonctionnalité de l'articulation coxo-fémorale naturelle (cartilage, ...) entraînent une 

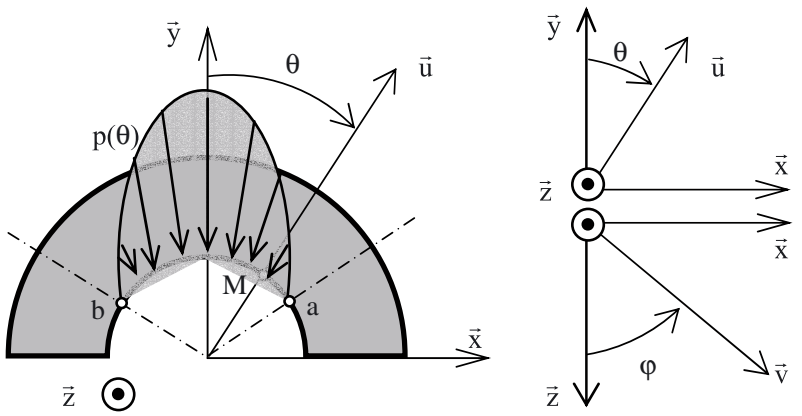

Fig. 8. Surface de contact entre tête de PTH et Cotyle.

Tableau 1. Caractéristiques mécaniques des biomatériaux utilisés.

\begin{tabular}{ccccc}
\hline Matériaux & $\begin{array}{c}\text { Module } \\
\text { de Young } \\
(\mathrm{GPa})\end{array}$ & $\begin{array}{c}\text { Coefficient } \\
\text { de } \\
\text { Poisson }\end{array}$ & $\begin{array}{c}\text { Limite } \\
\text { d'élasticité } \\
(\mathrm{MPa})\end{array}$ & $\begin{array}{c}\text { Masse } \\
\text { volumique } \\
\left(\mathrm{kg} \cdot \mathrm{m}^{-3}\right)\end{array}$ \\
\hline UHMWPE & 0,915 & 0,46 & 10,58 & 935 \\
$316 \mathrm{~L}$ & 200 & 0,30 & 284 & 7800 \\
$\mathrm{CoCrMo}$ & 230 & 0,30 & 440 & 9200 \\
$\mathrm{Al}_{2} \mathrm{O}_{3}$ & 320 & 0,27 & - & 3980 \\
\hline
\end{tabular}

déformation de la tête du fémur et du cotyle dans la région polaire caractérisée par l'apparition d'une aire de contact circulaire et aplatie.

\subsection{Modèle numérique}

Sur le plan mécanique, le choix des couples de matériaux, la technique d'ancrage à l'os et le design de la prothèse sont les principaux facteurs qui peuvent affecter les descellements aseptiques des implants articulaires.

\subsubsection{Matériaux utilisés}

Selon l'étude rétrospective [11], plusieurs modèles de PTH existent sur le marché, le modèle de Charnley semble être le plus utilisé à l'Institut M.T. Kassab. Les différents couples de matériaux fréquemment utilisés et faisant l'objet de cette étude sont le polyéthylène (UHMWPE) pour les cotyles, la céramique $\left(\mathrm{Al}_{2} \mathrm{O}_{3}\right)$ et l'acier inoxydable (316L) pour les têtes de PTH. Les propriétés mécaniques de ces matériaux diffèrent d'un auteur à un autre $[7,24-$ 26 ] et sont rassemblées dans le tableau 1. Le choix de tels couples de matériaux, reste encore tributaire de l'orientation des écoles d'orthopédie, mais arbitraire dans plusieurs cas.

Pour les besoins de l'étude, les calculs sont développés pour deux diamètres différents, le diamètre $\emptyset d=$ 22,2 mm, utilisé jusqu'aux années 1990 à l'Institut M.T. Kassab [27] et le diamètre $\emptyset d=28 \mathrm{~mm}$, utilisé actuellement de plus en plus dans beaucoup de centres orthopédiques publics et privés.
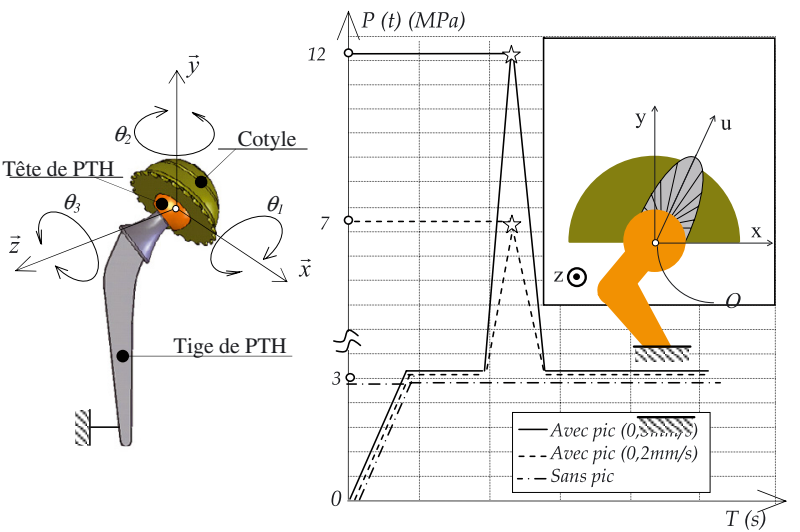

Fig. 9. Chargement appliqué sur PTH (a), conditions aux limites (b).

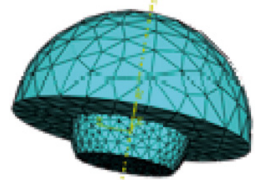

\begin{tabular}{ccc}
\hline Composant & $\begin{array}{c}\text { Type } \\
\text { d'élément }\end{array}$ & $\begin{array}{c}\text { Nombre } \\
\text { d'éléments }\end{array}$ \\
\hline Cotyle $28 \times 56$ & C3D4-Tétra & 1532 \\
Tête PTH $\emptyset 28$ & C3D4-Tétra & 6003 \\
\hline
\end{tabular}

Fig. 10. Exemple type de maillage de cotyle et tête de PTH $(\emptyset 22 \times 56)$.

\subsubsection{Conditions aux limites et de chargements et hypothèses de calculs}

Des études biomécaniques [28] montrent que la sollicitation physiologique au niveau de la liaison coxo-fémorale naturelle, peut varier de 3,5 à 5 fois le poids du sujet porteur de la prothèse. Pour une personne qui pèse $70 \mathrm{~kg}$, la charge moyenne appliquée à la tête de la PTH est de l'ordre de $3 \mathrm{MPa}$. Pour mettre en relief les effets des pics de chargement, nous avons imposé à l'articulation prothésée des vitesses de chargement variant de 0,2 à $0,5 \mathrm{~mm} . \mathrm{s}^{-1}$ et correspondant respectivement à des chargements de 7 et $12 \mathrm{MPa}$ (Fig. 9).

Dans la pratique un pic de chargement sur une hanche prothésée peut être induit, par exemple, suite à un saut rapide ou par le soulèvement d'une charge excessive.

Pour s'approcher au mieux des conditions de chargement physiologique, nous avons choisi l'axe passant par $(o, \vec{u})$ comme étant la direction principale de chargement et on admet qu'il existe un jeu radial uniforme $(0,05 \mathrm{~mm})$ entre la tête et le cotyle. La queue de la PTH est supposée encastrée et le cotyle est animé de trois rotations $(\mathrm{F} / \mathrm{E}$, $\mathrm{ADD} / \mathrm{ABD}$ et $\mathrm{RI} / \mathrm{RE})$.

Pour les deux composants étudiés (cotyle + tête), on a utilisé un mailleur standard et des éléments de type hexaédriques (Fig. 10).

\subsubsection{Démarche de calculs}

Les calculs sont développés sur prothèse hybride soumise à des pressions de contact avec et sans pic de chargement. Plusieurs paramètres mécaniques ont un effet direct sur l'élévation des contraintes prothétiques et le 


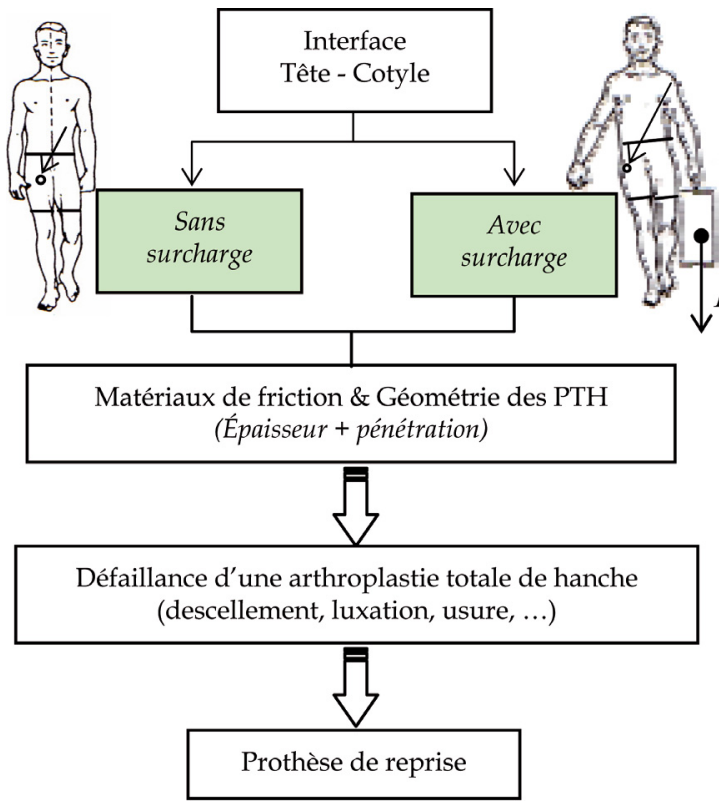

Fig. 11. Organigramme général de l'analyse numérique.

descellement de l'implant. À travers nos modèles de calculs nous cherchons à optimiser la résistance mécanique du système et le volume minimal du matériau propre au cotyle à implanter, tout en préservant un maximum de capital osseux. La pose d'un cotyle de diamètre externe élevé peut dans une certaine mesure, affecter la résistance et les mécanismes du remodelage de l'os (amincissement à travers l'épaisseur $\left(e_{\mathrm{c}}\right)$ de la surface d'appui sur la cavité de l'os coxal) (Fig. 6).

\section{Résultats et discussions}

Les principaux résultats de calculs portent sur les effets de la géométrie de l'implant (diamètre externe et interne), du type de chargement (avec et sans pic) et des couples de matériaux à travers leurs coefficients de frottement.

\subsection{Effet de la géométrie de la PTH (sans pic)}

La pose des PTH de première intention fait appel à des implants souvent du type standard (Tab. 2). La taille et les dimensions de la prothèse sont choisies selon les données de la radiologie sur le sujet admis pour une arthroplastie totale de hanche (ATH).

Selon l'étude rétrospective [11], nous montrons que les implants (cotyles) de diamètre $(d)$ égal à $22,2 \mathrm{~mm}$ sont couramment utilisés (prothèse de Charnley Kerboull). Nos premiers modèles de calculs portent sur une étude comparative de la contrainte de Von Mises pour une prothèse de diamètre interne 22,2 et $28 \mathrm{~mm}$ (Fig. 12).

Sous les mêmes conditions de chargement, la contrainte maximale de Von Mises passe de 3,8 MPa pour $\emptyset d=22,2 \mathrm{~mm}$ à $3,2 \mathrm{MPa}$ pour $\emptyset d=28 \mathrm{~mm}$. Ceci met en
Tableau 2. Dimensions des cotyles utilisés.

\begin{tabular}{ccccccccc}
\hline$d / D$ & \multicolumn{10}{c}{$\mathrm{D}(\mathrm{mm})$} \\
\hline$d(\mathrm{~mm})$ & 48 & 50 & 52 & 54 & 56 & 58 & 60 & 62 \\
\hline 22,2 & 0,46 & 0,44 & 0,42 & 0,41 & 0,39 & 0,38 & 0,37 & 0,35 \\
28 & 0,58 & 0,56 & 0,53 & 0,51 & 0,50 & 0,48 & 0,46 & 0,45 \\
32 & 0,66 & 0,64 & 0,61 & 0,59 & 0,57 & 0,55 & 0,53 & 0,51 \\
40 & 0,83 & 0,80 & 0,76 & 0,74 & 0,71 & 0,68 & 0,66 & 0,64 \\
\hline
\end{tabular}

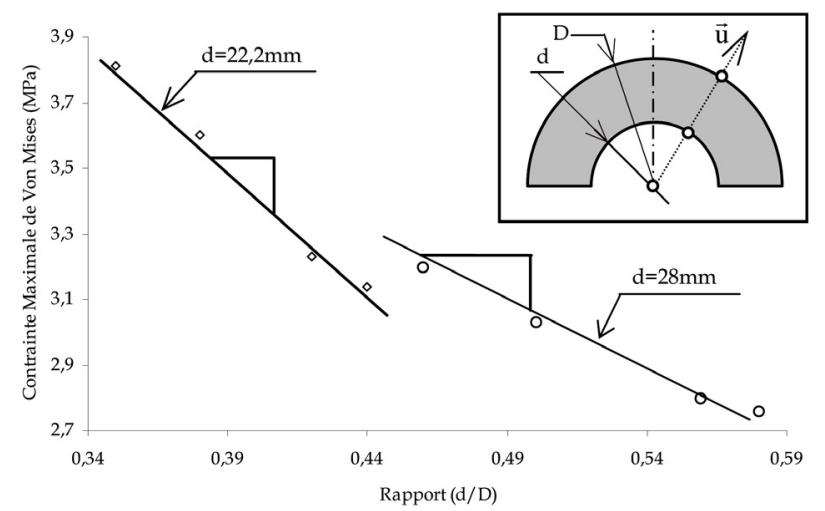

Fig. 12. Évolution de la contrainte de Von Mises maximale en fonction de l'épaisseur du cotyle pour $d=22,2 \mathrm{~mm}$ et $d=28 \mathrm{~mm}$.

relief l'importance de l'étendue de la surface de recouvrement sur la diminution des pressions et des contraintes prothétiques entre tête et cotyle de la PTH. Ce résultat préliminaire justifie l'intérêt des praticiens qu'ils portent pour les diamètres $28 \mathrm{~mm}$ au détriment des diamètres $22,2 \mathrm{~mm}$ pour la prothèse de hanche.

L'évolution des contraintes principales prises dans les plans de chargement $(o, \vec{u}, \vec{v})$, montre une prédominance des contraintes $\sigma_{11}$ et $\sigma_{33}$ lesquelles sont très actives respectivement dans les plans frontal et sagittal (Fig. 13). Ces résultats justifient les travaux de Bergmann [28] dont les résultats sont obtenus expérimentalement sur des prothèses instrumentées et montrent les limites du modèle de Pauwels [13]. La forte amplitude $\left( \pm 23^{\circ}\right)$ du mouvement perturbateur lors de la flexion/extension, a un effet amplificateur de contraintes mécaniques.

Plusieurs combinaisons de diamètres (internes et externes) pour cotyles sont calculées sous chargement sans pic. Dans la deuxième série de calculs, nous avons fixé le diamètre externe $(\emptyset D 56 \mathrm{~mm})$ et fait varier le diamètre interne de $\emptyset d 22,2$ jusqu'à $\emptyset d 40 \mathrm{~mm}$. D'un point de vue clinique, ceci revient à « amincir la couche» de l'implant (cotyle) en vue de préserver un maximum de capital osseux.

Nos calculs sur couple de matériaux UHMWPE/316L montrent que les évolutions des contraintes de Von Mises augmentent et tendent vers un état de contrainte plane (enveloppe mince) au fur et à mesure que le diamètre interne du cotyle augmente.

Dans la pratique chirurgicale, on cherche un compromis entre le «volume implanté » et la résistance mécanique de la prothèse. La combinaison $(\emptyset 28 \times 56 \mathrm{~mm})$ 


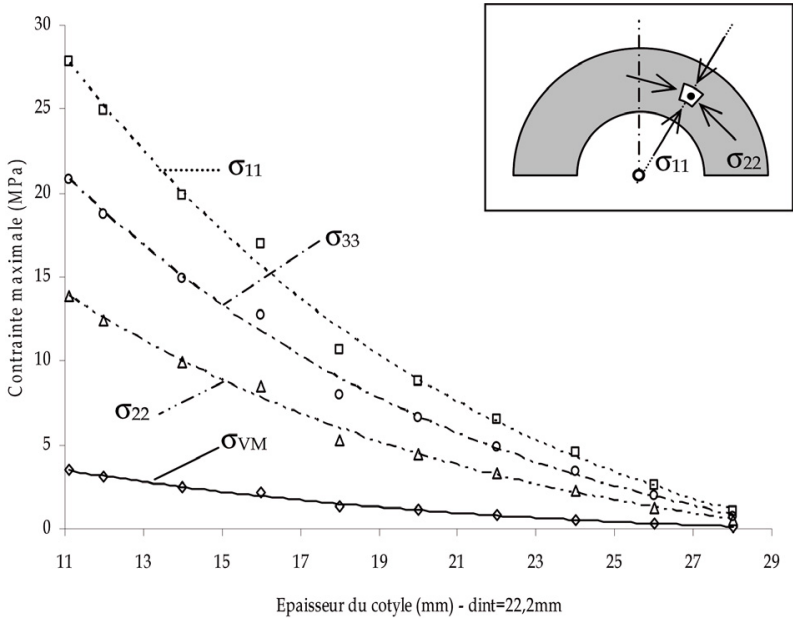

Fig. 13. Évolution des contraintes principales et de Von Mises en fonction de l'épaisseur du cotyle : $d=22,2 \mathrm{~mm}-$ sans pic de chargement - cas du couple UHMWPE/316L.

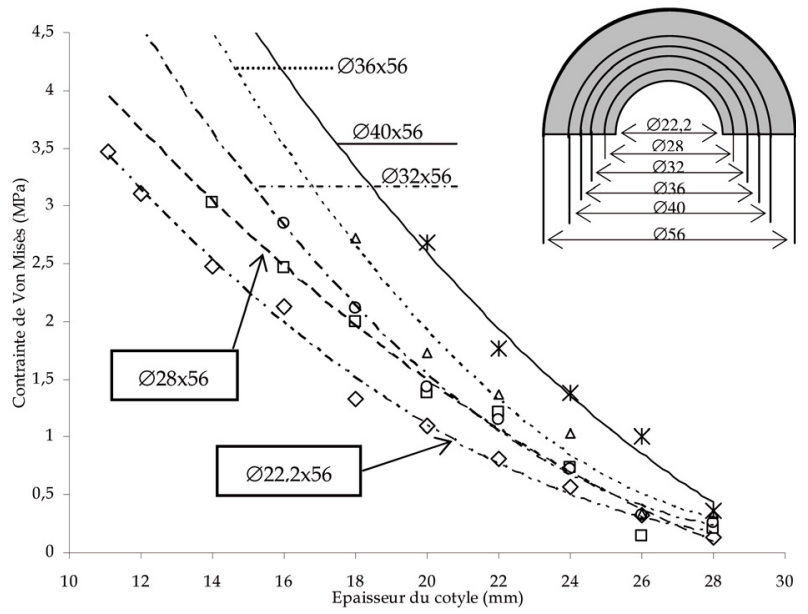

Fig. 14. Contrainte de Von Mises maximale - cas du couple UHMWPE / 316L.

semble satisfaire ce compromis [29] et justifie son adoption par beaucoup de chirurgiens $[7,27,30]$.

\subsection{Effet du chargement (avec et sans pic)}

Dans la troisième série de calculs, nous avons fixé le diamètre intérieur $\emptyset d 22,2 \mathrm{~mm}$ et fait varier le diamètre extérieur de $\emptyset D 48$ à $60 \mathrm{~mm}$ du cotyle, soumis aux conditions de chargements (avec et sans pic). L'analyse porte sur l'évolution de la contrainte de Von Mises pour le couple UHMWPE/316L.

Les contraintes enregistrées sous chargement avec pic sont élevées et atteignent des valeurs moyennes dix fois celles obtenues sans surcharge surtout au niveau de la surface de contact $(\emptyset d=22,2 \mathrm{~mm})$.

$\mathrm{Au}$ fur et à mesure que la vitesse de chargement croît, la contrainte de Von Mises augmente à proximité et au voisinage de la surface de contact mais on enregistre une

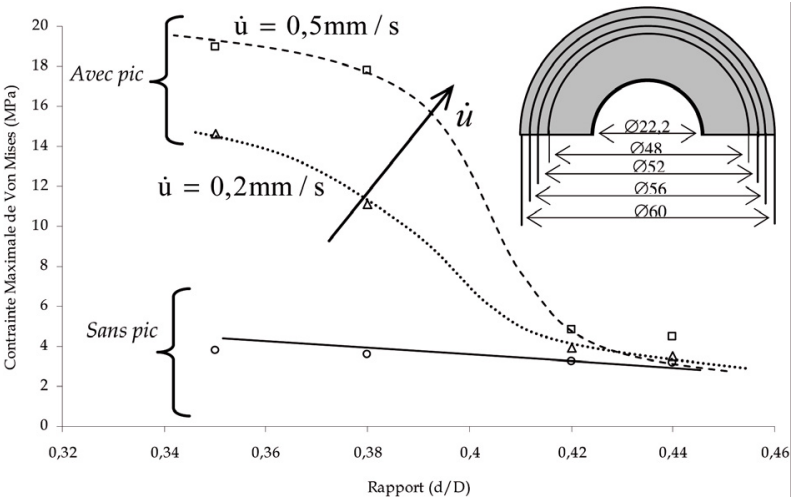

(a)
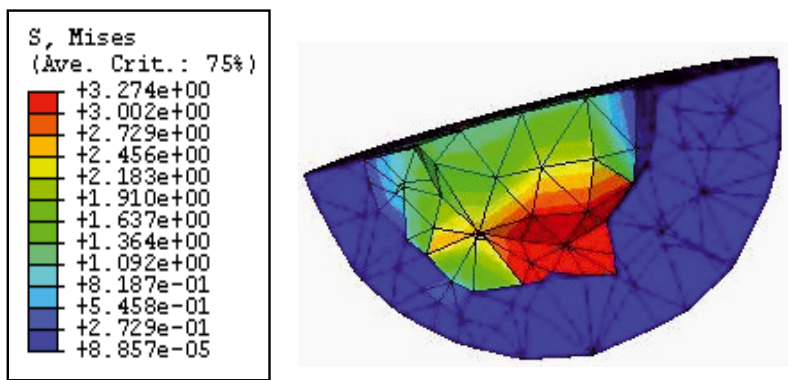

(b)
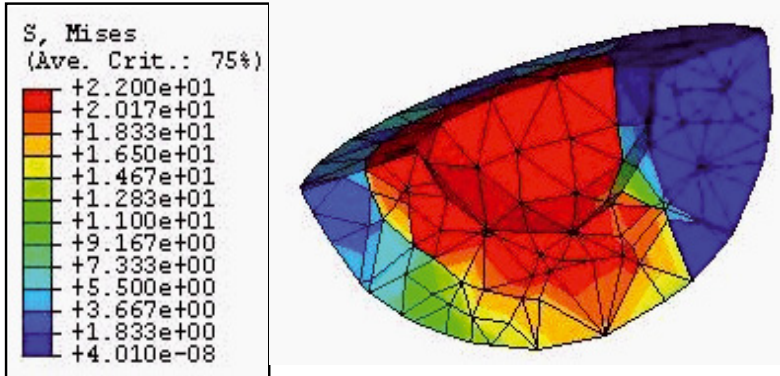

(c)

Fig. 15. (a) Évolution de la contrainte de Von Mises maximale en fonction de l'épaisseur du cotyle $-d=22,2 \mathrm{~mm}$. (b) Iso contrainte sur cotyle - cas sans pic de chargement. (c) Iso contrainte sur cotyle avec pic de chargement.

atténuation de la contrainte quand l'épaisseur du cotyle de l'implant augmente.

Dans la quatrième série de calculs, nous avons maintenu constant le diamètre extérieur du cotyle $(\emptyset D=$ $56 \mathrm{~mm}$ ) et varié son diamètre intérieur. Le chargement est fait avec pic à une vitesse de mise en charge $\dot{u}=$ $0,5 \mathrm{~mm} . \mathrm{s}^{-1}$.

Les résultats de calculs (avec pic) comparés à ceux obtenus sans pic, sont multipliés de 2,5 fois pour le diamètre $(\emptyset 40 \times 56 \mathrm{~mm})$ à 6 fois pour le diamètre $(\emptyset 22,2 \times 56 \mathrm{~mm})$ (Fig. 16). Les contraintes de Von Mises obtenues pour le diamètre $(\emptyset 28 \times 56 \mathrm{~mm})$ sont quatre fois plus élevées et constituent des valeurs intermédiaires situées entre les valeurs extrêmes du diamètre interne ( $\emptyset d 22,2$ et $40 \mathrm{~mm})$. Ce diamètre $(\emptyset 28 \times 56 \mathrm{~mm})$ est tolérable par la limite 


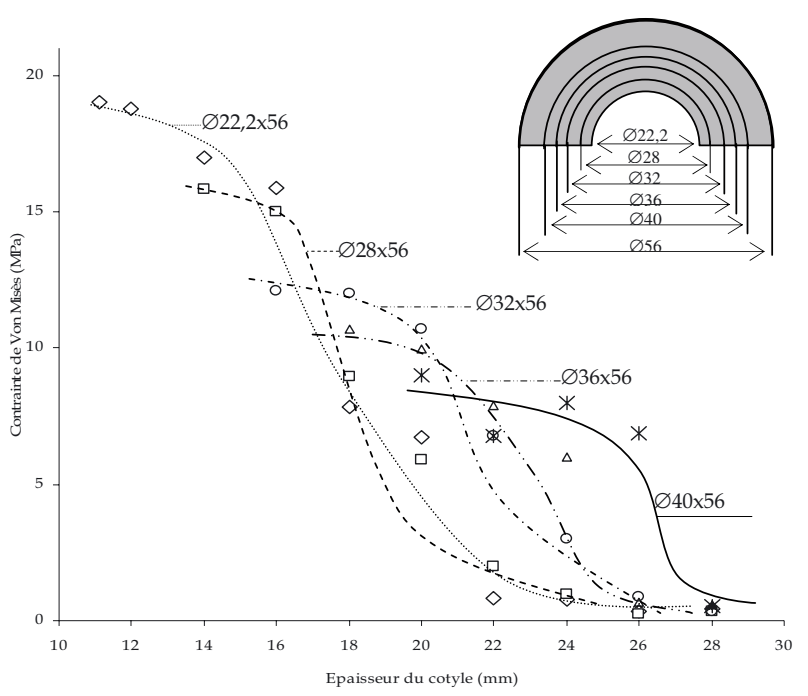

Fig. 16. Évolution de la pression maximale de contact en fonction de l'épaisseur du cotyle.

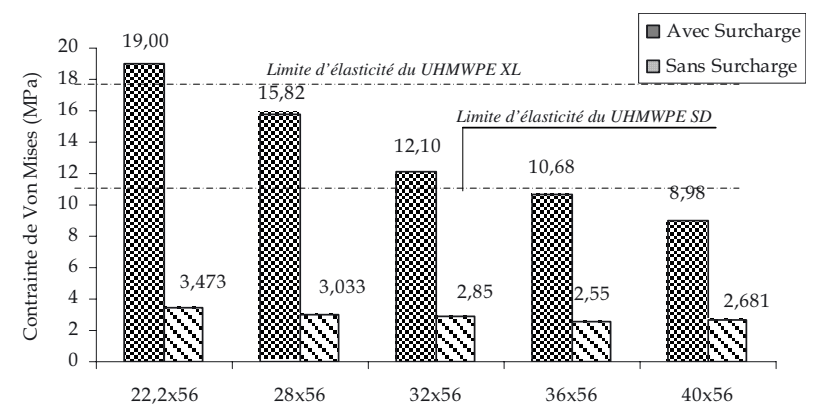

Fig. 17. Évolution de la contrainte de Von Mises maximale en fonction de l'épaisseur du cotyle, XL : hautement réticulé, SD : Standard.

du matériau testé selon la nuance choisie du polyéthylène (standard ou réticulé où $\sigma_{e}=11$ à $20 \mathrm{MPa}$ ).

Selon nos modèles de calcul, tous les résultats montrent les effets néfastes des pics de chargement sur la tolérance mécanique et la tribologie des matériaux prothétiques utilisés. Pour le couple UHMWPE/316L soumis aux mêmes conditions de chargement $(\dot{u}=$ $\left.0,5 \mathrm{~mm} . \mathrm{s}^{-1}\right)$, les rapports des contraintes de Von Mises, avec et sans pic, sont multipliés par trois pour le cotyle $\emptyset 40 \times 56 \mathrm{~mm}$ et par six pour le cotyle $\emptyset 22,2 \times 56 \mathrm{~mm}$ (Fig. 17).

À part les diamètres standards recommandés en chirurgie orthopédique (Ø28 $\mathrm{mm}$ pour le couple céramique/ céramique et $\emptyset 32 \mathrm{~mm}$ pour le couple métal/métal), l'utilisation des autres diamètres nécessite des matériaux de friction plus élaborés tels que le polyéthylène hautement réticulé (crosslinked) où la limite élastique est plus élevée que celle du polyéthylène haute densité [31] (Fig. 17).

Sous des conditions sévères de chargement avec pic $\left(\dot{u}=0,5 \mathrm{~mm} \cdot \mathrm{s}^{-1}\right)$, pour un cotyle de diamètre intérieur $\emptyset d=22,2 \mathrm{~mm}$, les contraintes principales atteignent des valeurs maximales qui dépassent nettement les contraintes admissibles par les matériaux

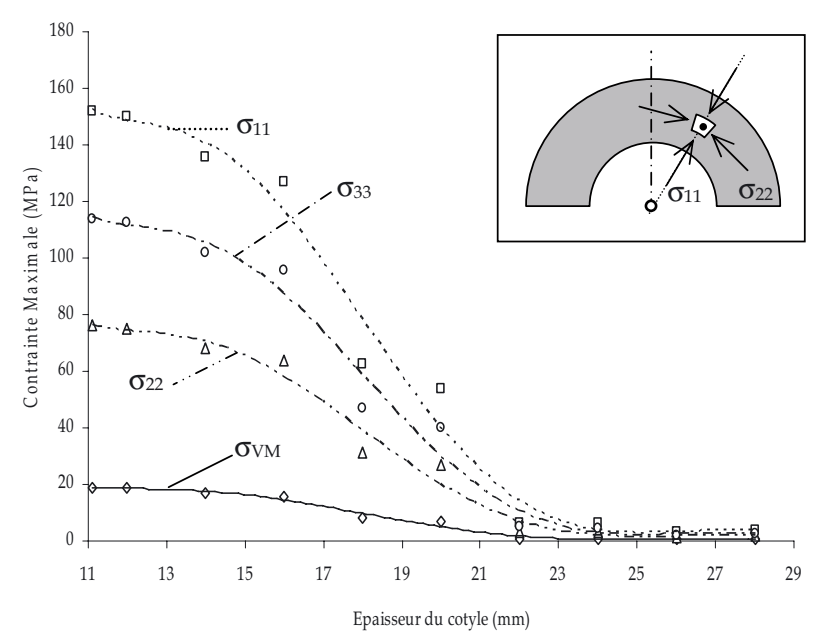

Fig. 18. Évolution des contraintes de Von Mises en fonction de l'épaisseur du cotyle : $d=22,2 \mathrm{~mm}$ - avec pic de chargement.

étudiés (Fig. 18). Physiquement ceci se traduit par une pénétration de la tête en acier inoxydable (316L) dans le polyéthylène (UHMWPE). Ce constat est vérifié sur plusieurs cotyles $(\emptyset 22,2 \mathrm{~mm})$ explantés et très fortement endommagés [2].

\subsection{Effet du couple de matériaux (avec et sans pic de chargement)}

Plusieurs couples de matériaux de friction sont utilisés en arthroplastie totale de hanche [7-10]. Dans la pratique, la tendance s'oriente vers les couples à faible taux de friction [23]. Dans cette série de calculs, nous comparons l'évolution des pressions de contact sur cotyle de diamètres $22,2 \mathrm{~mm}$ et $28 \mathrm{~mm}$ pour les couples $\left(\mathrm{UHMWPE} / \mathrm{Al}_{2} \mathrm{O}_{3}\right)$ et $(\mathrm{UHMWPE} / 316 \mathrm{~L})$ soumis à des sollicitations avec et sans pic de chargement.

Les calculs montrent en HPP, que la pression de contact augmente au fur et à mesure que le coefficient de friction croît. Cette pression est plus prononcée sur le diamètre 22,2 mm pour le couple (UHMWPE/ $/ \mathrm{Al}_{2} \mathrm{O}_{3}$ ). Ce résultat justifie l'absence sur le marché des implants articulaires PTH de diamètre de tête $\emptyset d 22,2 \mathrm{~mm}$ et fabriqués en céramique. Les têtes de diamètre $\emptyset d 28 \mathrm{~mm}$ comparées à celles de $22,2 \mathrm{~mm}$, favorisent des pressions de contact minimales allant du $1 / 10^{\mathrm{e}}$ jusque $1 / 2$ des valeurs nominales obtenues sur $d=22,2 \mathrm{~mm}$.

Sous chargement avec pic, la pression maximale dans les cas extrêmes $(d=22,2 \mathrm{~mm})$ peut être multipliée par dix pour des coefficients de frottement élevés (Fig. 21) et observés généralement après plusieurs années de service. L'emploi des biocéramiques (alumine et zircone), en dépit de leur bonne tenue à l'usure (faible friction), ne sont pas à l'abri des ruptures instables et prématurées sous l'effet des pics de chargement.

Le choix du type de la prothèse (PTH) dépend en grande partie des moyens disponibles (patient et centre orthopédique), mais aussi des expériences vécues 


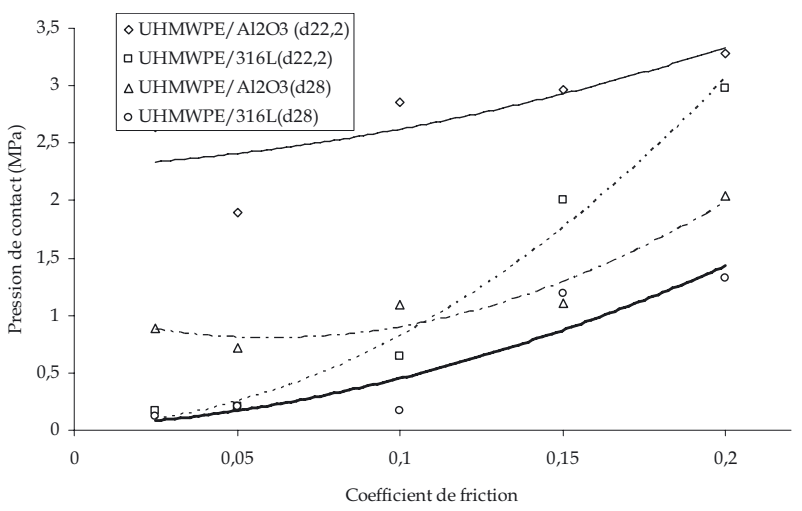

Fig. 19. Pression maximale de contact - sans pic de chargement.

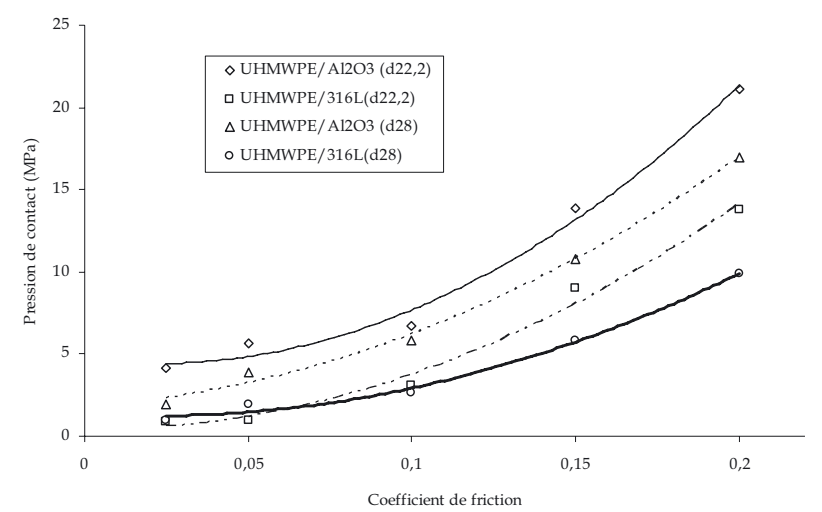

Fig. 20. Pression maximale de contact - avec pic de chargement.

par les différentes équipes chirurgicales. Certains services orthopédiques [30] préfèrent la prothèse $(\emptyset d 28 \mathrm{~mm})$ cimentée de couple de matériau céramique (tête)/ polyéthylène (cotyle).

D'après certains chirurgiens [32], ce choix favorise moins de pression, moins d'usure, un contact « amortissant » et moins rigide par rapport au contact céramique/céramique ou métal/métal et un coût relativement plus faible qu'une PTH «tout céramique ».

La dernière série de calculs porte sur une étude comparative sur prothèse de même diamètre $(\emptyset d 28 \mathrm{~mm})$ mais de deux matériaux de contact différents (UHMWPE/ $\mathrm{Al}_{2} \mathrm{O}_{3}$ ) et $\left(\mathrm{Al}_{2} \mathrm{O}_{3} / \mathrm{Al}_{2} \mathrm{O}_{3}\right)$. Les résultats de calculs montrent que l'évolution de la contrainte de Von Mises sur le couple $\left(\mathrm{Al}_{2} \mathrm{O}_{3} / \mathrm{Al}_{2} \mathrm{O}_{3}\right)$, atteint une valeur maximale de l'ordre de $270 \mathrm{MPa}$ proche de la limite de rupture de l'alumine (320 MPa en flexion). Le polyéthylène, sous mêmes conditions de chargement atteint une contrainte maximale de l'ordre de $18 \mathrm{MPa}$ (Fig. 21).

La nature du contact rigide offert par le couple $\left(\mathrm{Al}_{2} \mathrm{O}_{3} / \mathrm{Al}_{2} \mathrm{O}_{3}\right)$ fait que la contrainte de Von Mises atteint sa valeur maximale à la couche périphérique interne du cotyle de PTH jusqu'à une profondeur moyenne de l'ordre de $4 \mathrm{~mm}$ avant de chuter à $30 \mathrm{MPa}$.

Le même phénomène n'est pas observé sur le couple (UHMWPE $/ \mathrm{Al}_{2} \mathrm{O}_{3}$ ) où le contact est nettement moins rigide. La nature semi-cristalline du polyéthylène peut

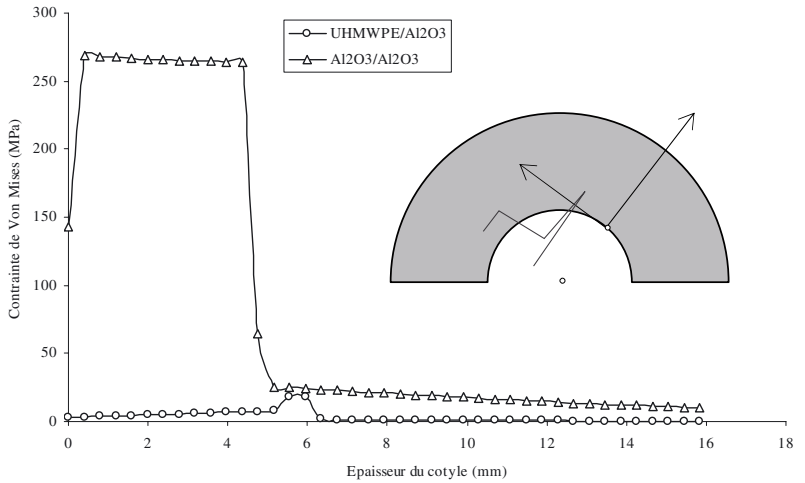

Fig. 21. Contrainte de Von Mises maximale $-\emptyset 28 \times 56$.

donner naissance à des contraintes résiduelles en volume et expliquer l'élévation des contraintes de Von Mises dans la direction de chargement et à une profondeur moyenne de $6 \mathrm{~mm}$.

L'aspect microstructural, très différent des biomatériaux utilisés (UHMWPE et $\mathrm{Al}_{2} \mathrm{O}_{3}$ ), peut expliquer cette différence [33]. En effet, pour les biocéramiques (matériaux durs et fragiles), le modèle des forces de liaisons inter-atomiques est analogue aux systèmes de ressort représentant une élasticité pure (linéaire ou non) et dépourvue de toute forme d'amortissement, les déformations se produisant à la même vitesse que les contraintes.

La dureté de la biocéramique, définie par la résistance à la pénétration de la tête dans le cotyle, peut expliquer ce résultat. Du point de vue physique, la pénétration met en jeu un ensemble de facteurs physico-chimiques difficiles à quantifier.

Les mécanismes de tensions en surface et de frottement, de déformation plastique et élastique entre couple de matériaux différents, interviennent lors de la pénétration de la tête dans le cotyle.

Dans le cas du polyéthylène, la compression provoque une dilatation latérale, les contraintes réelles seront donc différentes des valeurs nominales. L'écoulement visqueux (proportionnalité entre force et vitesse de déformation) caractérise un amortissement. Ainsi le comportement viscoélastique du polyéthylène peut être schématisé, en comparaison à la biocéramique, par un ressort symbolisant la force de liaison et un amortisseur qui contrôlera la vitesse de la déformation élastique. La contrainte transmise peut pour ce cas être atténuée (Fig. 21) à partir d'une certaine profondeur.

Sur le plan rhéologique, la déformation élastique s'accompagne souvent d'un frottement : c'est le frottement interne très présent dans les polymères thermoplastiques.

Pour le polyéthylène à structure ramifiée, des études de diffraction-X [33], ont révélé que l'axe des chaînes était perpendiculaire aux plaquettes cristallines. Puisque la longueur moyenne d'une chaîne est bien supérieure à l'épaisseur d'une plaquette, il faut émettre l'hypothèse que ces chaînes sont repliées régulièrement sur ellesmêmes (Fig. 22). 


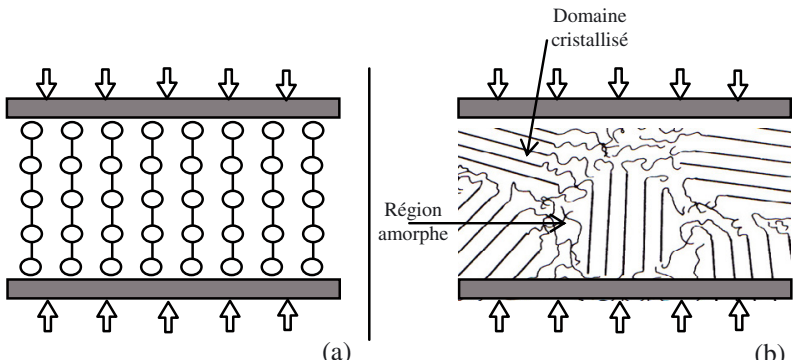

Fig. 22. Aspect microstructural de la biocéramique (alumine) (a) et polyéthylène (UHMWPE) (b).

Ainsi la rigidité du polyéthylène (matériau viscoélastique) est-elle directement fonction de la vitesse de déformation imposée? Celle-ci jouera également un rôle dans le domaine plastique de ce genre de matériau où le caractère visqueux est souvent plus accentué : l'augmentation de la consolidation apparente du matériau provoque une élévation des contraintes internes pouvant modifier complètement les mécanismes de déformation et d'amorçage de fissures de ces types de matériaux.

Dans un autre côté, ces contraintes après pic de chargement, peuvent être suffisamment élevées pour atteindre localement les contraintes de décohésion de la matrice. Les mécanismes de déformation et de rupture sont donc modifiés en cet endroit où la pression est très active (direction de la charge $\vec{R}$ ) et peuvent dans certains cas, transformer un matériau (statiquement) ductile en un matériau (dynamiquement) fragile.

\section{Conclusion}

Cette étude, axée sur la modélisation numérique des implants articulaires de hanche, met en relief le difficile compromis à respecter entre les observations cliniques et le comportement mécanique des PTH pour un chargement donné. Les résultats, les plus significatifs, de cette étude portent sur cinq points essentiels :

- une faible congruence de la prothèse, caractérisée par une surface de contact réduite (cas du diamètre $22,2 \mathrm{~mm}$ ), augmente la pression sur le polyéthylène d'autant plus que la charge appliquée est avec pic où la contrainte moyenne $\left(\sigma_{\mathrm{VM}}\right)$ est multipliée, à titre d'exemple, par sept pour $\dot{u}=0,5 \mathrm{~mm} . \mathrm{s}^{-1}$;

- les pressions et les contraintes engendrées par un dessin prothétique (PTH) peuvent être calculées en fonction de la géométrie de la prothèse (rayon, épaisseur) et du module d'élasticité du couple de matériaux utilisés. Les diamètres des PTH (interne et externe) incompatibles avec un niveau de pression acceptable, doivent être éliminés. Les autres peuvent être modélisés et testés par différentes méthodes (élémentsfinis [34], essais sur simulateurs [35], ...) avant d'être proposés pour l'utilisation clinique [36];

- lorsque les contraintes et les pressions de contact sont peu élevées, le mouvement relatif entre pièces de friction entraîne une usure abrasive lente et une usure adhésive et peut conduire à des descellements aseptiques de l'implant plus destructifs au fur et à mesure que l'état de surface de contact s'altère sous sollicitations de forte amplitude de chargement (pic);

- si le diamètre interne du cotyle doit être compatible avec des pressions tolérables par le couple de matériaux de friction sélectionné, son diamètre externe doit être bien optimisé pour éviter les résections osseuses importantes qui fragilisent le support osseux (cas des sujets âgés);

- comparativement au polyéthylène, l'alumine favorise des contacts rigides et des contraintes de contact très élevées. L'amélioration de la résistance du polyéthylène nécessite une plus grande fiabilité du procédé de fabrication et de contrôle, mais aussi de stérilisation. L'utilisation du polyéthylène réticulé en chirurgie orthopédique peut être une solution acceptable pour pallier aux problèmes de fragilité de l'alumine et au fluage du polyéthylène UHMWPE.

\section{Références}

[1] B. The, A. Hosman, J. Kootstra, V. Kralj-Iglic, G. Flivik, N. Verdonschot, R. Diercks, Association between contact hip stress and RSA-measured wear rates in total hip arthroplasties of 31 patients, J. Biomech. 41 (2008) 100-105

[2] A. Boulila, Optimisation et aide à la conception d'implants orthopédiques - Étude de l'usure d'implants articulaires de hanche, Travaux non publiés et rapport d'avancement, INO MT Kassab, Tunis, 82 p., décembre 2006

[3] M.R. Urist, Acrylic Cement Stabilized Joint Replacements, Curr. Prob. Surg. 12 (1975) 1-54

[4] A. Boulila, Étude expérimentale et modélisation numérique du gonflement hydraulique des plaques minces, DEA de Mécanique Appliquée Option Construction et Fabrication Mécanique, École Nationale d'Ingénieurs de Tunis, 2000, Tunisie

[5] J. Manuceau, Modèle à trois dimensions de l'articulation de la hanche, Rev. Chir. Orthop. 77 (1991) 293-300

[6] Ph. Frain, Hanche normale et prothétique - Un comportement de surface différent, Rev. Chir. Orthop. 69 (1981) 95-105

[7] D. Poitout, Biomécanique orthopédique, Éditions Masson, Paris New York, 1987

[8] S.M. Green, Deformation of materials, Curr. Orthop. 20 (2006) 9-15

[9] F. Langlais, Le polyéthylène en 1997, Les Cahiers de la SOFCOT, Pathologies de la hanche de l'adulte, pp. 37-51

[10] H. Baudriller, Approche micromécanique et modélisation de l'usure. Application aux biomatériaux de PTH, Thèse de doctorat en Mécanique, Université de la Méditerranée, décembre 2003, Marseille

[11] A. Boulila, K. Jendoubi, M. Douik, P. Chabrand, Étude des défaillances des arthroplasties totales de hanche, Congrès de la SOTCOT, Tunis, 2-3 Juin 2007

[12] O. Calonius, Tribology of prosthetic joints - Validation of wear simulation methods, Acta Polytechnica Scandinavica, Mechanical Engineering Series, $N^{\circ}$ 159, ESPOO 2002, p. 62 
[13] F. Pauwels, Biomechanics of the normal and diseased hip, Springer, Berlin, 1976

[14] N.W. Rydell, Forces acting on the femoral head prostheses: a study on strain gauge supplied prostheses on living persons, Acta Orthopedica Scandinavica 37 (1966) $1-132$

[15] J.P. Paul, Forces transmitted by joints in the human body, Proc. Inst. Mech. Eng. 8(3J) (1967) 181

[16] C.E. Carlson, A proposed method for measuring pressures on the human hip joint, Exp. Mech. 12 (1971) 499-506

[17] J.P. Paul, Influence of head constraint and muscle forces on the strain distribution within the intact femur, Med. Eng. Phys. 24 (2002) 243-249

[18] M. Nordin, V.H. Frankel, Basic biomechanics of musculoskeletal system, (3rd édition), Lippincott Williams and Wilkins, Baltimore (USA), 2001

[19] Ph. Frain, Évolution du vecteur gravitaire au cours de la marche normale - Corrélations musculaires et cinétiques, Rev. Chir. Orthop. 71 (1985) 537-547

[20] A. Boulila, K. Jendoubi, A. Zghal, P. Chabrand, Simulation numérique sur PTH du comportement tribologique du couple $\mathrm{PE} / 316 \mathrm{~L}$, Troisième Workshop AMINA'2006, ISBN : 978-9973 - 9963 - 3-3 Monastir, p. $115-117$

[21] E. Rixrath, S. Wendling-Mansuy, X. Flecher, P. Chabrand, J.N. Argenson, Design parameters dependences on contact stress distribution in gait and jogging phases after total hip arthroplasty, J. Biomech. 41 (2008) $1137-1142$

[22] S.M. Bouchard, K.J. Stewart, D.R. Peterson, J.J. Callaghan, T.D. Brown, Design factors influencing performance of constrained acetabular liners: finite element characterization, J. Biomech. 34 (2001) 859-871

[23] R.M. Hall, M.J.K. bankes, G. Blunn, Biotribology for joint human joints, Curr. Orthop. 15 (2001) 281-290

[24] S.H. Teoh, W.H. Chen, R. Thampuran, An elasto-plastic finite element for polyethylene wear in total hip arthroplasty, J. biomech. 35 (2002) 323-330

[25] R. Skalak, S. Chien, Handbook of bioengineering, ISBN 0-07-057783-8, McGraw, USA, 1987
[26] R. Sakai, M. Itomoan, K. Mabuchi, Assessements of different kinds of stems by experiments and FEM analysis: appropriate stress distribution on a hip prostheses, Clin. biomech. 21 (2006) 826-833

[27] M. Douik, Apport de la biomécanique et des biomatériaux avancés pour la chirurgie orthopédique, point de vue des orthopédistes, $3^{\text {es }}$ Journées de biomécanique, biomatériaux avancés et Imagerie Médicale, Tunis 14-16 mai 2001

[28] G. Bergmann, G. Deurtzbacher, M. Heller, F. Graichen, A. Rohlmann, J. Strauss, G.N. Duda, Hip contact forces and gait patterns from routine activities, J. Biomech. 34 (2001) 859-871

[29] L. Sedel, L'alumine en chirurgie orthopédique, Les cahiers d'enseignement de la Sofcot, $\mathrm{N}^{\circ} 25$ Biomatériaux en chirurgie orthopédique - Expo-Scie Française

[30] A. Khelil, K. Jendoubi, L. Nouisri, Le couple Polyéthylène/alumina en arthroplastie totale de hanche : le point de vue clinique et scientifique, Congrès de la SOTCOT, Tunis 2-4 juin 2007

[31] J.G. Browsher, J.C. Shelton, A hip simulator study of influence of patient activity level on the wear of crosslinked polyethylene under smooth and roughened femoral conditions, Wear 250 (2001) 167-179

[32] Maîtrise Orthopédique, www.maitrise-orthopedique. com

[33] J.P. Baïlon, J.M. Dorlot, Des Matériaux, $3^{\mathrm{e}}$ édition, École Polytechnique de Montréal, 2000, ISBN 2-553-00770-1

[34] J. Shih-Shyn Wu, Jui-Pin Hung, Chi-shiang Shu, JianHorng Chen, The computer simulation of wear behaviour appearing in total hip prostheses, Comput. Methods Programs Biomed. 70 (2003) 81-91

[35] O. Calonius, V. Saikko, Slide track analysis of eight contemporary hip simulator designs, J. Biomech. 35 (2002) 1439-1450

[36] J.P. Delagoutte, D. Mainard, M. Dupuy, G. Peltre, C. G'Sell, Étude des descellements et de l'usure des prothèses totales de hanche - À propos d'une série de plus de 10 ans de recul, Rev. Chir. Orthop. 77 (1991) $25-33$ 